\title{
Lipid-Derived Mediators are Pivotal to Leukocyte and Lung Cell Responses in Sepsis and ARDS
}

\author{
Julie Nijmeh ${ }^{1} \cdot$ Bruce D. Levy $\mathbb{D}^{1}$ \\ Received: 24 May 2021 / Accepted: 4 June 2021 / Published online: 27 June 2021 \\ (c) The Author(s), under exclusive licence to Springer Science+Business Media, LLC, part of Springer Nature 2021
}

\begin{abstract}
Acute inflammation in the lung is essential for host defense against pathogens and other injuries but chronic or excessive inflammation can contribute to several common respiratory diseases. In health, the inflammatory response is controlled by several cellular and molecular mechanisms. In addition to anti-inflammatory processes, there are non-phlogistic proresolving mechanisms that are engaged to promote the resolution of inflammation and a return to homeostasis. Defects in the production or actions of specialized pro-resolving mediators are associated with diseases characterized by excess or chronic inflammation. In this article, we review cellular and biochemical mechanisms for specialized pro-resolving mediators in health and in sepsis and the acute respiratory distress syndrome as examples of unrestrained inflammatory responses that result in life-threatening pathology. We are honored to contribute to this special edition of the Journal to help celebrate Professor Viswanathan Natarajan's contributions to our understanding of lipid-derived mediators and metabolism in lung cell responses to inflammatory, infectious, or mechanical insults; his foundational discoveries in cell biochemistry and biophysics are continuing to catalyze further advances by the field to uncover the mechanistic underpinnings of important human diseases.
\end{abstract}

Keywords Inflammation $\cdot$ Resolution $\cdot$ Specialized pro-resolving mediators $\cdot$ Neutrophils $\cdot$ Phospholipase D $\cdot$ Presqualene diphosphate

$\begin{array}{ll}\text { Abbreviations } \\ \text { ALI } & \text { acute lung injury } \\ \text { ARDS } & \text { acute respiratory distress syndrome } \\ \text { BAL } & \text { bronchoalveolar lavage } \\ \text { CF } & \text { cystic fibrosis } \\ \text { Cif } & \text { conductance regulator inhibitory factor } \\ \text { COPD } & \text { chronic obstructive pulmonary disease } \\ \text { CysLT } & \text { cysteinyl leukotriene } \\ \text { HCl } & \text { hydrochloric acid } \\ \text { ICU } & \text { intensive care unit } \\ \text { IDP } & \text { isodielectric point } \\ \text { IDS } & \text { isodielectric separation } \\ \text { LXA } & \text { lipoxin A } 4 \\ \text { MaR1 } & \text { maresin 1 } \\ \text { Mo-PA } & \text { monocyte-platelet aggregate } \\ \text { Ne-PA } & \text { neutrophil-platelet aggregate }\end{array}$

$\begin{array}{ll}\text { NETs } & \text { neutrophil-extracellular traps } \\ \text { PA } & \text { phosphatidic acid } \\ \text { PI3K } & \text { phosphatidylinositol 3-kinase } \\ \text { PIPP } & \text { polyisoprenyl phosphates } \\ \text { PLPP } & \text { phospholipid phosphatase } \\ \text { PLD } & \text { phospholipase D } \\ \text { PSDP } & \text { presqualene diphosphate } \\ \text { PSMP } & \text { presqualene monophosphate } \\ \text { ROS } & \text { reactive oxygen species } \\ \text { RvD1 } & \text { resolvin D1 } \\ \text { RvD2 } & \text { resolvin D2 } \\ \text { RvE1 } & \text { resolvin E1 } \\ \text { SPM } & \text { specialized pro-resolving mediator } \\ \text { VILI } & \text { ventilator-induced lung injury }\end{array}$

\section{Introduction}

Acute inflammatory responses are essential to health for protection from environmental insults such as pathogens, allergens, pollutants, and injuries [1]. Acute inflammation is initiated within seconds after challenge. This vital process is normally self-limited with an initiation and resolution phase 
culminating with the restoration of tissue homeostasis. This process of returning an inflamed tissue back to a homeostatic baseline has been termed "catabasis" [2]. Impaired engagement of acute inflammation can lead to tissue injury; however, recent evidence indicates that ineffective resolution can delay or disrupt catabasis, also resulting in tissue injury from unrestrained and prolonged inflammation. In the lung, unrestrained immune responses of several types are associated with clinical diseases, including asthma, chronic obstructive pulmonary disease, cystic fibrosis (CF), and acute respiratory distress syndrome (ARDS) [3] (Table 1).

Resolution of acute inflammation is an active response that is mediated by select resolution phase mediators [4]. There are several families of specialized pro-resolving mediators (SPMs) that are generated during multicellular host inflammatory responses [5]. SPMs are agonists of specific pro-resolving receptors that are expressed in celltype specific manners. SPM-activated cellular effectors function to limit inflammation and restore tissue homeostasis and function (Table 1). At the tissue or organism level, there are five essential resolution parameters (the five "R"s), namely "R"emoval of inflammatory cells and tissue debris, "R"estoration of vascular integrity and perfusion, "R"egeneration and repair of tissue, "R"emission of fever, and "R"elief from inflammatory pain [4]. SPMs mediate each of these resolution parameters that together comprise the process of catabasis.

SPMs are derived by enzymatic conversion of essential dietary fatty acids. Structurally distinct families of SPMs are classified by their fatty acids origins, including lipoxins (LXs) from arachidonic acid (C20:4, n-6); E-series resolvins from eicosapentaenoic acid (EPA, C20:5, n-3); and D-series resolvins, protectins, and maresins from docosahexaenoic acid (C22:6, n-3) [6] (Fig. 1). Interestingly, several epidemiological studies show an inverse correlation between diets rich in omega-3 fatty acids and the prevalence of inflammatory diseases $[7,8]$. In addition, omega-3 fatty acid supplementation during pregnancy can be protective in children for symptomatic wheeze and respiratory infections [9, 10]. SPMs are rapidly produced for local actions and rapidly inactivated, exhibiting potent receptor-mediated spatial and temporal regulation of inflammatory responses in the lung. SPMs are functionally defined by their dual actions as anti-inflammatory "stop" signals for neutrophils and as pro-resolving "go" signals for macrophage phagocytosis and efferocytosis. As part of a larger resolution program, defects in the SPM pathway have been identified in several inflammatory diseases [4].

In this article, we review roles for SPMs in infectious immunity and inflammatory pulmonary diseases, and explore their signaling mechanisms that control cellular responses to inflammatory stimuli. Here, in this special issue of the Journal in dedication to his retirement, we also shed light on the many contributions of Professor Viswanathan Natarajan's pioneering research on lipid-derived signaling mechanisms for lung cell activation and control.

\section{Sepsis and ARDS}

In health, a self-limited inflammatory response to an infection can successfully contain and clear the pathogen and restore homeostasis and health. An overwhelmed or dysregulated immune response can result in systemic inflammation, organ dysfunction, shock and, in the extreme, death, such as can occur in sepsis [11-13]. Notably, sepsis is one of the principal risk factors for ARDS, a complex syndrome characterized by such exuberant lung inflammation that the alveolar-capillary membrane is disrupted with consequent pulmonary edema and lung injury that leads to significant and potentially life-threatening hypoxemia [14]. Despite advances in clinical care and some improvement in clinical outcomes, both sepsis and ARDS remain devastating illnesses with high mortality and morbidity and still no host-targeted treatments, highlighting the need for a deeper understanding of the cellular and molecular basis of sepsisinduced systemic immune dysregulation [11-13].

At the center of the early inflammatory response to sepsis and ARDS is the recruitment and activation of leukocytes to the site of infection or injury; however, excessive leukocyte activation can be maladaptive and contribute to tissue inflammation and organ damage [15]. The infiltrating leukocytes respond to multiple environmental and endogenous signals that dictate their activation [16]. Given the sensory role of leukocytes, their function and activation state are indicative sentinels of the host response to sepsis progression or resolution and may serve as predictors of illness severity. Monitoring of leukocyte counts alone is too variable and not a reliable predictive measure of sepsis clinical responses for individual patients. In experimental sepsis in mice, organ injury and the severity of the infection can be reduced by SPMs (i.e., resolvin D2) that regulate leukocyte activation [17]. To more sensitively monitor leukocyte activation, electrical label-free cell profiling of leukocytes by isodielectric separation was recently developed to determine the membrane isodielectric point (IDP) of neutrophils as a biomarker of their activation state [18]. The neutrophil IDP changes early in murine sepsis concomitant with cell activation (as confirmed by flow cytometry parameters) [18]. This new technology was recently tested in human sepsis [19]. For preparative leukocyte isolation from small volumes of peripheral blood, an innovative sample sparing closed-loop inertial microfluidic platform was developed [19]. This inertial microfluid separation enables label-free isolation of adequate leukocyte numbers from microliter blood volumes for ascertainment of cell IDP. 

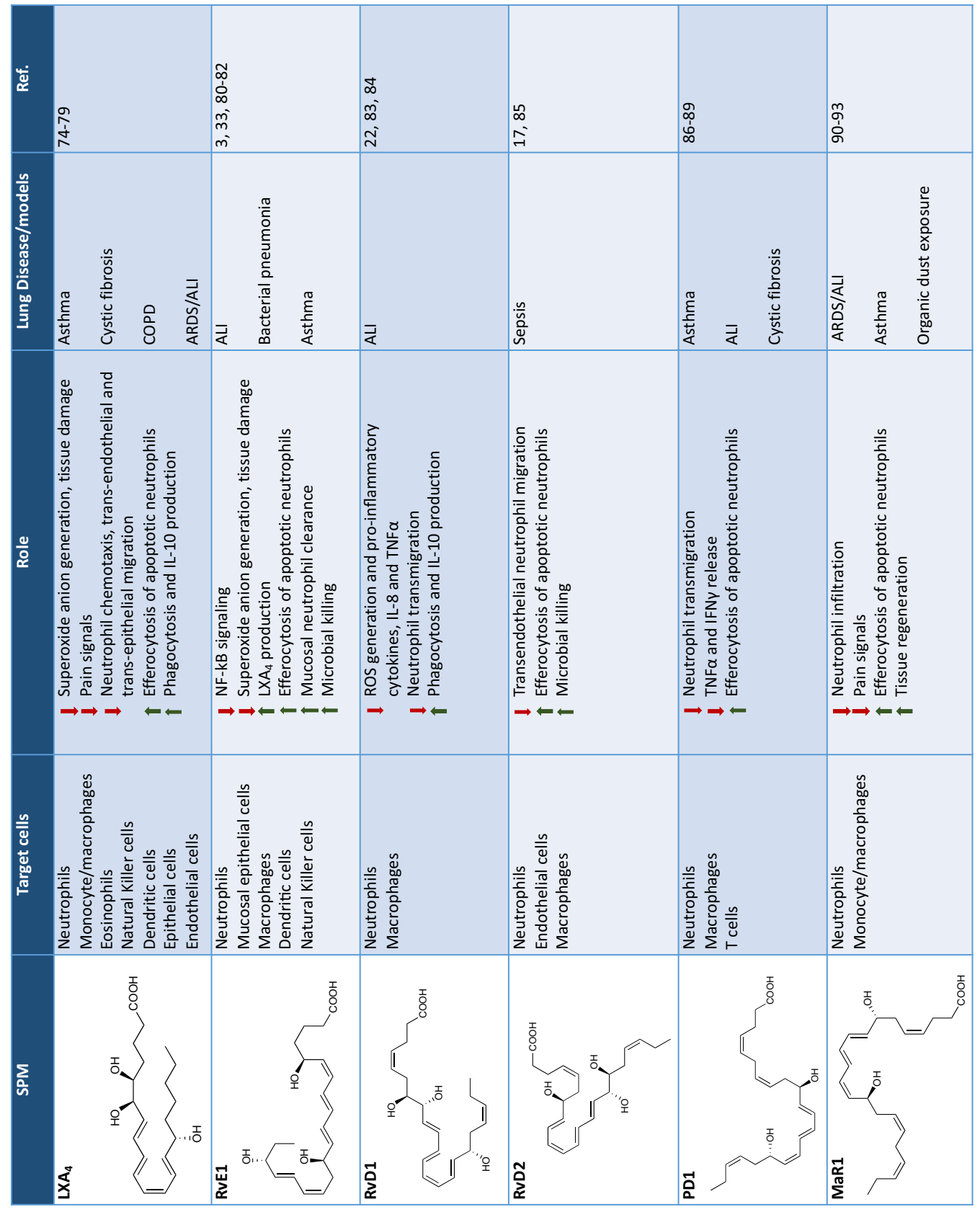


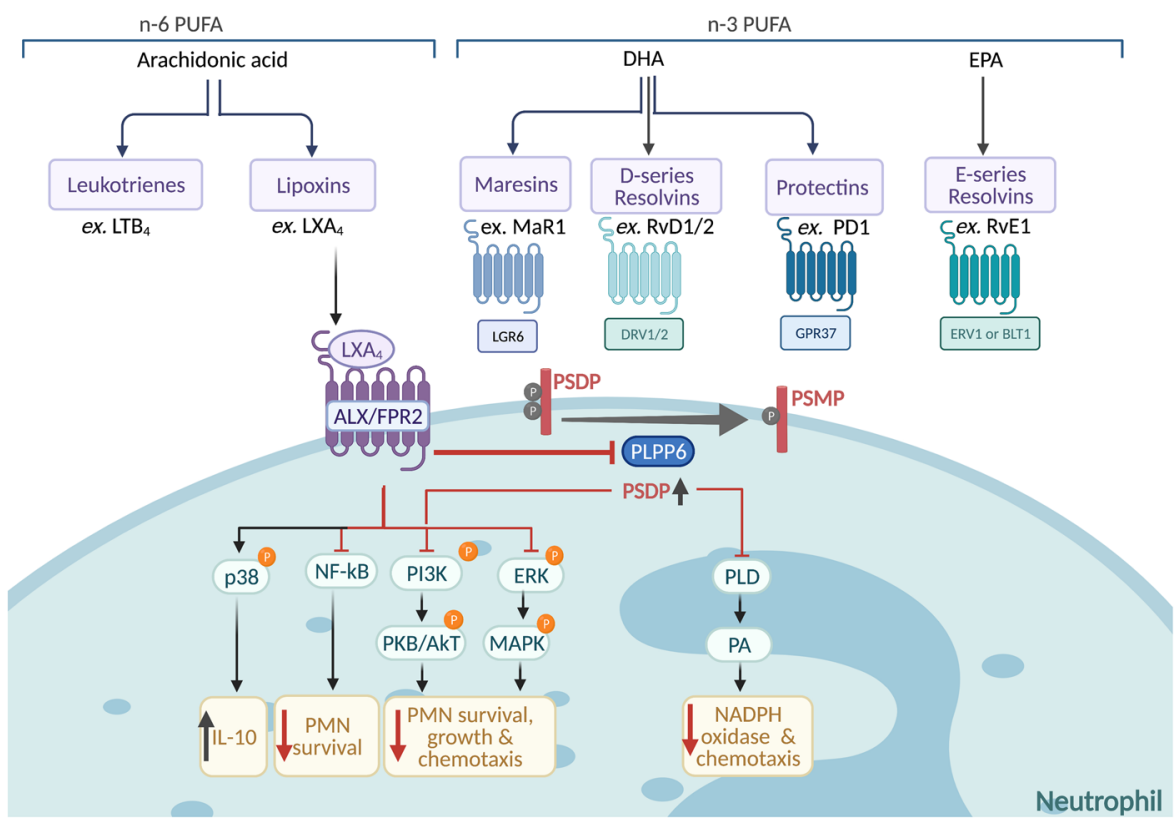

Fig. $1 \mathrm{SPMs}$, their receptors, signaling and actions in neutrophils. Simplified biosynthesis pathway of the SPM families namely lipoxins, maresins, resolvins, and protectins with examples of identified receptors. Downstream signaling pathways and polyisoprenyl phosphate remodeling in neutrophils upon $\mathrm{LXA}_{4}-\mathrm{ALX}$ interactions: $\mathrm{LXA}_{4}$ acts as a "stop signal" during inflammation to inhibit PLD, PI3K, NFkB

Because this device is sample sparing, the risk for iatrogenic anemia, a common problem in intensive care units (ICU), is reduced [19]. This sample sparing approach allows for repeated assessment of the neutrophil IDP as a biomarker of the host response to monitor disease progression for critical illnesses such as sepsis and ARDS in humans. This new approach opens the possibility for a better understanding of how early biochemical and biophysical events in sepsis and ARDS influence leukocyte activation and may ultimately help clinicians monitor disease severity and support the development of new preventive and treatment strategies.

There are many pro-inflammatory mediators such as cytokines and chemokines that activate leukocytes to propel the host inflammatory response to pathogens or tissue injury [20]. There are a similarly large array of endogenous mediators produced to counterregulate the pro-inflammatory signals as part of an active resolution program that promotes a return to homeostasis [4, 5]. Principal among these counterregulatory signals are the SPMs. These autacoid lipid-derived mediators have dual anti-inflammatory and pro-resolving properties $[4,5]$. During self-limited acute inflammatory responses SPMs are produced during the resolution phase. Of note, select SPMs, such as resolvins and LXs, can reduce leukocyte-mediated lung tissue damage $[21,22]$. Many experimental studies have shown that SPM restraint of acute inflammatory processes leads to timely resolution and mitigation of innocent bystander tissue damage [22,23]. Of interest, lipid mediator metabolomes are among others and block PSDP turnover to PSMP. PSDP inhibits PLD and PI3K to further reduce pro-inflammatory responses. Red lines represent inhibition, and black arrows represent activation of a pathway. PMN, polymorphonuclear neutrophils. Signaling schematic was adapted from Bonnans et al. and Krishnamoorthy et al. [4, 73]. Created with BioRender.com

dysregulated in sepsis with peripheral blood lipid mediator profiles correlating with survival, respiratory failure and ARDS development [24]. For example, lower plasma levels of select SPMs (i.e., lipoxin $\mathrm{A}_{4}\left(\mathrm{LXA}_{4}\right)$ and maresin 1 (MaR1)) and concomitantly higher levels of prophlogistic lipid mediators (i.e., cysteinyl leukotrienes (CysLTs) and thromboxane) are associated with increased duration of ventilatory support and ICU length of stay [25]. Individualized CysLTs to SPM ratios that are increased are indicative of increased lung inflammation, which is seen in most patients with ARDS; and was associated with increased disease severity and worse clinical outcomes in this study [25]. SPM profiling has the potential to shed light on patient status in critically ill subjects and sets the stage for SPM signature profiling of biosynthetic pathway activation as novel biomarkers for evaluating outcomes and represent biologic therapeutic targets for modulating treatment responses in sepsis.

In a sub-study of the Lung Injury Prevention Study with Aspirin (LIPS-A) clinical trial, the relationships between peripheral white blood cells and lipid mediators was determined in patients at high risk for the development of ARDS [26]. In this study, peripheral blood neutrophils, monocytes, leukocyte-platelet aggregates, and select bioactive lipid mediators were assessed and related to clinical development of ARDS. Intravascular leukocyte and platelet activation is integral to early inflammatory responses in ARDS, leading to heterotypic neutrophil-platelet 
aggregate (Ne-PA) and monocyte-platelet aggregate (MoPA) formation. These cell aggregates leverage secondary capture of leukocytes by platelet adhesion molecules that engage activated endothelial cells, leading to targeted and enhanced leukocyte tissue entry and tissue inflammation [27]. These events are regulated in part by lipid mediators: platelet released thromboxane promotes leukocyte adhesion to endothelial cells [28], whereas the SPM LXA 4 and its aspirin-triggered epimer 15-epi-LXA $\mathrm{LA}_{4}$ blunt leukocyte activation [21, 29, 30]. Of interest, blocking Ne-PA formation decreases experimental ARDS [23]. Results from the LIPS-A clinical trial sub-study demonstrated that circulating Ne-PA were elevated in these high risk patients irrespective of ARDS development [26]. Of note, Mo-PA numbers at enrollment were associated with progression to ARDS, supporting a pivotal role for intravascular cellular and lipid mediator events in early ARDS pathogenesis [26].

\section{Neutrophil Activation in Critical Illness}

\section{Pneumonia and Host Responses}

One of the most common causes of sepsis and ARDS is acute pneumonia, which is a leading cause of excess global morbidity and mortality [31]. Pneumonia pathobiology is characterized by neutrophil activation that is important for microbial host defense but can also lead to lung injury [32]. To limit lung tissue damage, similar to ARDS, neutrophil responses need to be restrained. In a murine model of nosocomial pneumonia from $E$. coli, the SPM resolvin E1 (RvE1) decreased neutrophil accumulation in the lung while concomitantly enhancing microbial clearance and survival [33]. This SPM is enzymatically derived from EPA and when given after the development of pneumonia can decrease neutrophil migration and pro-inflammatory cytokines (including IL-1 $\beta$, IL-6, MIP-1 $\alpha$, MIP-1 $\beta, \mathrm{KC}$, and MCP-1) while enhancing microbial clearance from the lung [33]. RvE1 also plays a pivotal role in host defense against Pseudomonas aeruginosa, a gram negative bacterium linked to acute exacerbations of lung diseases, including CF [34].

CF patients are more susceptible than healthy individuals to pneumonia from pulmonary infections with Pseudomonas aeruginosa [35]. The $P$. aeruginosa CF transmembrane conductance regulator inhibitory factor (Cif) is a secreted epoxide hydrolase, so it can disrupt transcellular biosynthesis of 15-epi $\mathrm{LXA}_{4}$, which is a potent regulator of neutrophil activation and tissue inflammation. Of note, bronchoalveolar lavage (BAL) fluid samples from $\mathrm{CF}$ patients with elevated levels of Cif have lower levels of 15-epi $\mathrm{LXA}_{4}$, as well as increased levels of the inflammatory cytokine IL-8, and impaired lung function [34]. The observation that Cif levels correlated with more severe disease in CF patients and that Cif targets endogenous epoxide containing molecules involved in inflammation and resolution, suggests that it could serve as a potential therapeutic target [36]. A small molecule targeting approach of Cif would result in blocking the enzymatic degradation of lipid mediators that together promote resolution of inflammation by reducing neutrophil infiltration, bacterial burden, and overall lessening disease severity.

\section{Acute Lung Injury (ALI) and Host Responses}

In addition to pathogen-initiated inflammation in pneumonia, tissue injury can lead to acute lung inflammation [37]. SPMs also display protective actions in this experimental setting. In a self-limited mouse model of hydrochloric acid ( $\mathrm{HCl}$ )-induced ALI that mirrors clinical acid aspiration, lung cyclooxygenase- 2 is induced and contributes to the early acute inflammation and late induction of 15lipoxygenase for LX production and resolution [21]. This self-limited murine model of acid-induced ALI has been useful for studying the biochemistry and immunology of lung inflammation resolution [38]. In addition to LXs, other SPMs can contribute to the resolution of ALI-evoked inflammation, including MaR1 and resolvins [22, 23]. The SPM actions include promoting the clearance of alveolar edema, increasing the efferocytosis of apoptotic neutrophils by recruited macrophages, and accelerating the reepithelialization of the airways and alveoli to reduce vascular leakage and tissue hypoxia [3].

Non-infectious organ injury can also occur in the setting of transplant rejection. Lung allograft rejection is associated with increased BAL fluid $\mathrm{LXA}_{4}$ levels [39]. In mouse models of solid organ transplantation, $\mathrm{LXA}_{4}$ signaling via its cognate receptor ALX/FPR2 protects against allograft rejection [39]. When an $\mathrm{LXA}_{4}$ analog is administered in organ perfusate and by the intravenous route at the time of transplantation, organ rejection was decreased in two separate and independent experimental models of vascularized transplantation [39]. In both HCl-initiated ALI and acute allograft rejection, SPM regulation of neutrophil recruitment and activation was essential to SPM conveyed tissue protection.

\section{NETosis in Severe Asthma}

One cellular response of extreme neutrophil activation is the process of neutrophil extracellular trap formation, termed NETosis. NETosis leads to the release of DNA-containing NETs from cells [40]. In many situations NETosis results in cell death; however, in some cases the enucleated cells can reseal to form cytoplasts. This cellular response for neutrophils occurs in severe pneumonia [40], including SARS 
CoV-2 infection [41], and was recently linked to the pathogenesis of severe asthma in a subset of patients with non-type 2 inflammation [42]. These patients with severe "neutrophilic" asthma have increased neutrophil counts in their BAL with detectable cytoplasts in patients with $>5 \%$ BAL neutrophils and increased BAL fluid IL-17 levels [42]. This subset of asthma patients with increased BAL neutrophils and NETosis had increased risk for asthma comorbidities linked to infections, including frequent exacerbations and sinusitis. In addition, some severe asthma patients have evidence for NETosis in their sputum with increased extracellular hyper-citrullinated DNA and increased IL- $1 \beta$ levels indicating inflammasome activation in their airways [43]. The phenomenon of vital NETosis with neutrophil cytoplasts was replicated in a mouse model of LPS exposure during allergen sensitization with house dust mite extract (a common household allergen) [42]. The neutrophil cytoplasts engaged lung dendritic cells to promote differentiation of naive CD4+ $\mathrm{T}$ cells to Th17 CD4+ T cell effectors, resulting in allergen-mediated Th17driven immune responses. These findings shed light on novel treatment possibilities, such as SPMs, for asthma associated with excess neutrophil recruitment, activation, and NETosis.

\section{SPMs and Regulation of Neutrophil Activation}

As reviewed above and in multiple additional studies, SPMs as a class of mediators carry potent in vivo regulatory properties that restrain neutrophil activation (Fig. 1). Of note, at a cellular level, neutrophil function is not entirely blocked by SPMs; rather they are modulated, which is important for preservation of some of their host protective actions [44]. Eicosanoids of different classes are structurally and functionally distinct. Most leukotrienes (LTs) and prostaglandins (PGs) contribute to acute inflammation, including neutrophil chemotaxis, degranulation, and superoxide release. In contrast, LXs counter the LT and PG proinflammatory actions to serve as "stop signals" for neutrophil trafficking and inflammatory responses.

SPMs play crucial roles in the resolution of lung mucosal inflammation in a myriad of conditions. RvE1 is important in the resolution of allergic airway inflammation, and 15-epi$\mathrm{LXA}_{4}, 17-\mathrm{epi}-\mathrm{RvD} 1$, and MaR1 are protective during $\mathrm{HCl}$ acid-induced lung injury $[6,21,22]$. In addition to these sterile inflammatory insults, in a murine model of pathogeninitiated lung inflammation, 17-epi-RvD1 and RvE1 also mediate host protection [33, 45]. During self-limited E. coli pneumonia, 17-epi-RvD1 levels are increased concomitant with resolution [45]. This temporal regulation of SPM levels was also associated with increased numbers of lung macrophages that can produce and respond to SPMs. 17-epiRvD1 stimulated macrophages in a non-phlogistic manner to promote phagocytosis of bacteria, and efferocytosis of apoptotic neutrophils, and also enhanced lung expression of lipocalin-2, an anti-microbial siderophore that limits the growth of E. coli [45]. Together, these anti-inflammatory, anti-microbial, and pro-resolving mechanisms were additive to antibiotics in lessening the severity and duration of this model of nosocomial pneumonia.

In addition to apoptotic neutrophils being cleared by macrophage efferocytosis, neutrophil clearance can also proceed via reverse migration (i.e., retrotaxis) away from sites of inflammation [46]. The lipid mediator $\mathrm{PGE}_{2}$ is released by macrophages following neutrophil engulfment in efferocytosis and plays several important roles in resolution, including a key role in lipid mediator class switching to SPMs. $\mathrm{PGE}_{2}$ switches lipid mediator biosynthesis from predominantly pro-inflammatory $\mathrm{LTB}_{4}$ to pro-resolving $\mathrm{LXA}_{4}$ via EP4 receptor signaling and cAMP-mediated CREB activation of 15-lipoxygenase expression for LX production [44]. $\mathrm{PGE}_{2}$ and $\mathrm{LXA}_{4}$ then serve to repel neutrophils so that they migrate away from the site of infection [46], possibly by interfering with potential "retention signals" that retain neutrophils in place.

Another important counterregulatory action for SPMs is to decrease pro-inflammatory cytokine production (e.g., BAL fluid TNF- $\alpha$ and MIP-2 after ALI) in part via inhibition of NF- $\mathrm{KB}$ in lung epithelial cells and macrophages [22]. SPM regulates NF- $\kappa B$ via A20 and SIGRR, potent intracellular inhibitors of NF- $\mathrm{KB}$ activation [47]. Of interest, bone marrow-derived mesenchymal stem cells, through cell-cell interactions with human alveolar epithelial type II cells, can increase lung $\mathrm{LXA}_{4}$ levels and upregulate ALX/ FPR2 expression under inflammatory stimulation and as such may leverage the endogenous SPM signaling pathways to enhance resolution [48].

\section{Polyisoprenyl Phosphate Signaling Regulates Neutrophil Activation}

In response to environmental stimuli, leukocyte membrane remodeling generates biologically active lipid mediators that depending on timing and signal transduction can dictate cellular responses [49]. To control cellular responses during acute inflammation it is critical to regulate leukocyte activation. In a search for intracellular regulators of leukocyte activation, screening of neutrophil-derived extracts revealed a fraction rich in phosphorylated, non-saponifiable lipids that carried regulatory actions for phosphatidic acid (PA)initiated NADPH oxidase assembly and superoxide anion generation in a cell-free system [50]. Physical chemical analyses led to the structural elucidation of four main polyisoprenyl phosphates (PIPPs) in this regulatory fraction of neutrophil lipids, namely farnesyl diphosphate (FDP), 
Table 2 Presqualene diphosphate (PSDP): evidence for roles as a signaling molecule

- Rapid and reciprocal remodeling of PSDP to PSMP upon neutrophil activation by soluble pro-inflammatory stimuli [50]

- PLPP6 (a.k.a. PDP1); a lipid phosphate phosphatase that converts PSDP to PSMP [55]

- Inhibition of PLD and subsequent PA production [51, 52]

- Inhibition of PI3K [53]

- Inhibition of NADPH oxidase assembly and superoxide anion production in neutrophils $[50,51]$

- SPMs (i.e., $\mathrm{LXA}_{4}$ ) inhibit PLPP6 and PSDP conversion to PSMP [55]

- Protective action of PSDP can be replicated by synthetic polyisoprenyl phosphate mimetics [53]

farnesyl monophosphate (FMP), presqualene diphosphate (PSDP), and presqualene monophosphate (PSMP) [50]. PSDP is present in unactivated cell membranes and in response to receptor-mediated pro-inflammatory stimuli, PSDP is rapidly and reciprocally remodeled to PSMP within seconds. PSDP, but not PSMP, acts as a potent inhibitor of NADPH oxidase assembly, as well as phospholipase D (PLD) [51, 52] and phosphatidylinositol 3kinase (PI3K) activity [53], thus PSDP can limit neutrophil superoxide anion $\left(\mathrm{O}_{2}^{-}\right)$production, degranulation, and chemotaxis [54] (Fig. 1).

A lipid phosphate phosphatase that converts PSDP to PSMP was identified as a member of the phospholipid phosphotransferase/phosphatase (PLPP) family of enzymes and is now named PLPP6 (aka PDP1, PPAPDC2, CSS2) [55-57]. Distinct from the parent member of this family PLPP1, PLPP6 only converts the isoprenyl diphosphate (i.e., PSDP or FDP) to its monophosphate form (i.e., PSMP or FMP) whereas PLPP1 will also convert the isoprenyl monophosphate to the corresponding alcohol (e.g., farnesol) [56]. It is notable that PSDP is produced by squalene synthase from two molecules of FDP, then PSDP can be converted by squalene synthase in an NADPH-dependent second step reaction to squalene as a biosynthetic intermediate for cholesterol [56]. In isolated enzyme systems, PSDP production by squalene synthase is available for conversion by PLPP6 to PSMP. Thus, PSDP production does not inevitably result in squalene biosynthesis; rather it is available for additional signaling functions as described here (Table 2). Several pro-inflammatory soluble stimuli for neutrophils activate PLPP6 for PIPP remodeling with PLPP6 phosphorylation by phospho-PKC $\beta$ II playing an important role in its activation [54]. SPMs appear to transduce their "stop signaling" for neutrophils, in part, by regulation of PKC $\beta I I$ phosphorylation, thereby preventing PLPP6 activation and enabling increased cellular levels of PSDP that can inhibit PLD and PI3K activity and NADPH oxidase [55, 58] (Fig. 1). PIPP remodeling provides a signaling mechanism for SPM receptor-mediated transduction of cell-type-specific responses. PLPP6 is the first identified phosphatase for PSDP, yet it can also dephosphorylate other substrates, most notably FDP [56, 57]. PLPP6 is expressed in human leukocytes and multiple human tissues and upon exposure to $\mathrm{LTB}_{4}$ is activated to decrease PSDP levels, suggesting that PLPP6 is a potential new therapeutic target to dampen overexuberant neutrophil activation in disease [53]. PLPP6 activity can be reversed by an ALX/FPR2 receptor agonist, such as 15-epi-LXA ${ }_{4}$ that also inhibits $\mathrm{LTB}_{4}$-initiated PLD and PI3K activation and superoxide anion generation [51, 53, 54]. These findings provide evidence for receptor-initiated PIPP remodeling as a regulatory signaling pathway. Novel synthetic PIPP mimetics, such as (Z)-containing isomer of PSDP ((Z)PSDP), have been synthesized and carry potent inhibition for experimental models of neutrophil accumulation, activation and leukocyte-driven reactive oxygen species (ROS) generation, toward controlling inflammatory responses and limiting tissue injury [52].

\section{PLD and ARDS}

The airway epithelium, in addition to being the first line of defense as a physiochemical barrier, plays an essential role in production and secretion of inflammatory mediators to augment host defense in the lungs [59]. Close communication between the pulmonary vascular endothelium and respiratory epithelium is important for coordination of the recruitment, activation, and retention of leukocytes during inflammation [60].

In addition to its important roles in leukocyte activation, PLD signaling is engaged in activation of lung epithelium. Multiple isoforms of PLD exist, of which PLD1 and PLD2 are enzymatically active and have been implicated in human pathophysiology, including ARDS [61]. PLD catalyzes the hydrolysis of the membrane phospholipid, phosphatidylcholine, generating choline and PA, which in turn regulates multiple intracellular signaling responses, including activation of $\mathrm{NF \kappa B}$ and activator protein-1 transcription factors resulting in airway epithelial cell secretion of the pro-inflammatory cytokine IL-8 [59]. IL-8 is a potent chemoattractant and activator of neutrophils, with reports of elevated levels in the BAL fluid of patients with a variety of respiratory disorders including ARDS [62].

PLD isoforms are differentially associated with survival in human patients with ARDS, and differentially regulate mouse host responses to ALI [61]. This has been highlighted via a targeted analysis of PLD and PLD-related gene expression of a published human gene expression microarray dataset in patients with ARDS. PLD1 gene expression was increased in patients with ARDS and correlated with 
survival, whereas PLD2 expression was associated with mortality. Upon comparison of gene expression between an animal model of ALI and human ARDS, the changes with lung inflammation were comparable in both species with an association between gene expression and recovery from disease [61]. In a murine model of self-limited ALI, PLD1 gene expression increased in injured lungs, and PLD1 deficiency in a knockout mouse worsened ALI severity by alveolar barrier disruption and decreased macrophage phagocytosis. In contrast, PLD2 expression decreased in murine self-limited ALI and PLD2 deficiency in a knockout mouse lessened ALI severity, and was associated with increased recruitment of macrophages with enhanced phagocytosis and decreased neutrophil production of ROS [61]. Together, these findings have uncovered pathogenic and protective signaling circuits of PLD1 and PLD2 that regulate the intensity of inflammation and its resolution thus influencing the nature and severity of lung injury and inflammation; emphasizing the potentially important role of PIPP remodeling and its effect on PLD activity for a controlled inflammatory response without progression to ARDS [61].

\section{Dr. Natarajan's Contributions to Lung Cell Biochemistry and Lipidology}

It is an honor for us to participate in this special issue of the Journal that is dedicated to Professor Natarajan and his lifelong passionate investigation of lipid biochemistry and its translation to cell biology and important lung diseases. His basic research on lipid phosphates and their phosphatases, especially sphingosine-1-phosphate (S1P) and its corresponding phosphatases, have uncovered several mechanisms for intracellular lipid signaling control of fundamental processes in lung cell biology [63-71]. Dr. Natarajan showed the important role of sphingosine in pulmonary endothelial cell signal transduction. Of note, sphingosine directly, or indirectly (upon conversion to S1P), can activate PLD, leading to accumulation of PA with downstream cellular responses [72]. In addition, sphingosine (but not S1P) can also inhibit PA phosphatase activity (i.e., the enzyme converting PA to diacylglycerol). PLDmediated PA generation plays an important role as a bioactive lipid second messenger in the regulation of various cellular processes including cell survival, cell migration, cell proliferation, differentiation, cytoskeletal changes, membrane trafficking, and autophagy [61].

Pathogen-induced lung inflammation is characterized by excess ROS generation [65]. Dr. Natarajan's lab identified a key role for NOX4 in mediating Pseudomonas-induced lung inflammation and injury via nuclear ROS generation, chromatin remodeling (by oxidant inhibition of HDAC1/2 activity) and secretion of pro-inflammatory cytokines [65]. $P$. aeruginosa instillation into mouse airways stimulates $\mathrm{NF}-\kappa \mathrm{B}$ in epithelial cells, and S1P signaling regulates NOX4-dependent nuclear ROS via Rac1. These novel results laced together a signaling cascade for $\mathrm{PA}$-induced PKC with downstream activation of SPHK2, Rac1, and NOX4, leading to nuclear ROS generation [65]. Furthermore, NOX4 was pivotal for epithelial apoptosis and differentiation of fibroblasts to myofibroblasts in lung fibrosis mouse models. Together, these studies highlight the potential targeting of NOX4 in mitigating lung infection and other pulmonary inflammatory diseases.

Patients with sepsis or pneumonia can develop respiratory failure resulting in the need for assisted ventilation [14]. Overinflation of the lung or extended duration of mechanical ventilation can cause ventilator-induced lung injury (VILI). Using a mouse model of VILI, Dr. Natarajan's group identified decreased levels of S1P in lung tissues, which correlated with increased expression of S1P Lyase (S1PL; an enzyme that converts S1P to $\Delta 2$-hexadecenal and ethanolamine phosphate) [66]. Of note, there is suggestive evidence that 2-halofatty aldehydes metabolites of S1P, derived from its degradation by S1PL, can serve as signaling lipids in mammalian cells [68]. Because mechanical ventilation in mice was associated with increased S1PL expression and consequent reduction of S1P levels, the corresponding signaling pathways could be important contributing mechanisms to alveolar epithelial cell apoptosis, barrier dysfunction as well as inflammatory cytokine release in VILI.

Regeneration and repair of tissue are integral to resolution of lung injury and inflammation. Dr. Natarajan's group has identified an interesting and important role for S1P signaling in this process by its regulation of the regenerative function of alveolar type 2 (AT2) cells, which are important for restitution of the integrity of the alveolar epithelial barrier. In a mouse model of bacterial lung injury, angiocrine S1P via the S1P-S1PR2-YAP signaling axis promotes differentiation of AT2 to AT1, thus regulating alveoli epithelial repair [64]. This insightful work highlighted the importance of endothelial cell communication and interaction with epithelial cells for lung barrier integrity and repair mediated by bioactive S1P autacoid signaling.

Together, Dr. Natarajan's fundamental contributions to our understanding of lysophospholipid and lipid phosphate phosphatase biochemistry in lung cell responses to inflammatory, infectious, or mechanical insults have uncovered physiologic and pathologic mechanisms for cell activation and control. His impactful discoveries have had a particular focus on heterotypic cell-cell interactions for regulation of alveolar epithelial barrier homeostasis and its restitution after injury during catabasis. These findings have also provided insights into fibroblast biology and interstitial fibrosing 
diseases. The potentially broad impact of Dr. Natarajan's science underscores its fundamental importance.

\section{Concluding Remarks}

In summary, here we have reviewed evidence in support of important roles for lipid-derived mediators in cellular responses in the pathobiology of sepsis and ARDS. Proinflammatory prostanoids and LTs are potent chemoattractants and activating mediators for leukocytes and tissue resident cells. Sepsis is a clinical syndrome of lifethreatening systemic inflammation. Similarly, ARDS is a syndrome of excess inflammation in the lung that compromises oxygenation and typically requires support with a mechanical ventilator that places patients at further risk for VILI. Both of these critical illnesses are propelled, in part, by vigorous and unrestrained leukocyte activation by proinflammatory lipid mediators. In addition to the upslope of inflammatory responses, in health, lipid mediators are also potent regulators of the resolution of these inflammatory responses. SPMs are enzymatically derived from essential polyunsaturated fatty acids at sites of inflammation and serve as receptor-mediated autacoids to resolve the inflammatory response and restore homeostasis. Pro-inflammatory stimuli, including LTs, activate PIPP remodeling to transiently unlock leukocyte cellular responses; signal transduction pathways that are blocked by resolution phase mediators, including LXs. In addition to PIPPs, other phosphorylated lipids, such as S1P, can regulate cell activation in inflammatory responses. In addition to leukocyte responses, lipid mediators regulate lung structural cell barrier integrity and regeneration and repair of injured tissues -restorative functions for the resolution of lung infection, trauma, and environmental insults. The study of lipidderived mediators and metabolism has contributed much to our understanding of acute lung injury and systemic inflammation, yet there are still many questions to answer. Contributions of leaders like Professor Natarajan have paved a scientific path forward to address these questions and have mentored a generation of scientists to carry the mission forward for an even better understanding of the underlying lipid biochemistry and cell biology of sepsis and ARDS toward the development of new therapeutics for these critical illnesses.

Author Contributions B.D.L. conceived the idea of the article, J.N. wrote the first draft of the manuscript, including the figure and tables, J.N. and B.D.L. performed the literature search, drafted, and critically revised the work.

Funding This work was supported in part by National Institutes of Health P01GM095467, U24 AI118656, and 1U01HL146002 (to B.D.L.).

\section{Compliance with Ethical Standards}

Conflict of Interest B.D.L. is an inventor on patents (SPMs) assigned to Brigham and Women's Hospital. J.N. has no conflicts of interest to declare that are relevant to the content of this article.

Publisher's note Springer Nature remains neutral with regard to jurisdictional claims in published maps and institutional affiliations.

\section{References}

1. Nathan, C., \& Ding, A. (2010). Nonresolving inflammation. Cell, $140,871-882$

2. Serhan, C. N. (2014). Pro-resolving lipid mediators are leads for resolution physiology. Nature, 510, 92-101

3. Levy, B. D., \& Serhan, C. N. (2014). Resolution of acute inflammation in the lung. Annual Review Physiology, 76, 467-492

4. Basil, M. C., \& Levy, B. D. (2016). Specialized pro-resolving mediators: Endogenous regulators of infection and inflammation. Nature Review Immunology, 16, 51-67

5. Serhan, C. N., \& Levy, B. D. (2018). Resolvins in inflammation: Emergence of the pro-resolving superfamily of mediators. Journal of Clinical Investigation, 128, 2657-2669

6. Krishnamoorthy, N., Abdulnour, R. E., Walker, K. H., Engstrom, B. D., \& Levy, B. D. (2018). Specialized proresolving mediators in innate and adaptive immune responses in airway diseases. Physiological Reviews, 98, 1335-1370

7. Albert, C. M., et al. (2002). Blood levels of long-chain n-3 fatty acids and the risk of sudden death. The New England Journal of Medicine, 346, 1113-1118

8. Galli, C., \& Rise, P. (2009). Fish consumption, omega 3 fatty acids and cardiovascular disease. The science and the clinical trials. Nutrition and Health, 20, 11-20

9. Bisgaard, H., et al. (2016). Fish oil-derived fatty acids in pregnancy and wheeze and asthma in offspring. The New England Journal of Medicine, 375, 2530-2539

10. Ramaswami, R., Serhan, C. N., Levy, B. D., \& Makrides, M. (2016). Fish oil supplementation in pregnancy. The New England Journal of Medicine, 375, 2599-2601

11. Delano, M. J., \& Ward, P. A. (2016). Sepsis-induced immune dysfunction: Can immune therapies reduce mortality? Journal of Clinical Investigation, 126, 23-31

12. Shen, X. F., Cao, K., Jiang, J. P., Guan, W. X., \& Du, J. F. (2017). Neutrophil dysregulation during sepsis: An overview and update. Journal of Cellular and Molecular Medicine, 21, 1687-1697

13. Singer, M., et al. (2016). The Third International Consensus definitions for sepsis and septic shock (Sepsis-3). JAMA, 315, 801-810

14. Baron, R. M., \& Levy, B. D. (2016). Recent advances in understanding and treating ARDS. F1000Res, 5, F1000 Faculty Rev-725

15. Weiss, S. J. (1989). Tissue destruction by neutrophils. The New England Journal of Medicine, 320, 365-376

16. Majno, G., \& Joris, I. (2004). In Cells, tissues, and disease: Principles of general pathology (chap. 8, 2nd, ed.). Oxford University Press, 307-332

17. Spite, M., et al. (2009). Resolvin D2 is a potent regulator of leukocytes and controls microbial sepsis. Nature, 461, 1287-1291

18. Prieto, J. L., et al. (2016). Monitoring sepsis using electrical cell profiling. Lab on a Chip, 16, 4333-4340

19. Jundi, B., et al. (2019). Leukocyte function assessed via serial microlitre sampling of peripheral blood from sepsis patients correlates with disease severity. Nature Biomedical Engineering, Emphasis Type="Italic">3, 961-973 
20. Dinarello, C. A. (2010). Anti-inflammatory agents: Present and future. Cell, 140, 935-950

21. Fukunaga, K., Kohli, P., Bonnans, C., Fredenburgh, L. E., \& Levy, B. D. (2005). Cyclooxygenase 2 plays a pivotal role in the resolution of acute lung injury. The Journal of Immunology, 174, 5033-5039

22. Eickmeier, O., et al. (2013). Aspirin-triggered resolvin D1 reduces mucosal inflammation and promotes resolution in a murine model of acute lung injury. Mucosal Immunology, 6, 256-266

23. Abdulnour, R. E., et al. (2014). Maresin 1 biosynthesis during platelet-neutrophil interactions is organ-protective. Proceedings of the National Academy of Sciences of the United States of America, $111,16526-16531$

24. Dalli, J., et al. (2017). Human sepsis eicosanoid and proresolving lipid mediator temporal profiles: Correlations with survival and clinical outcomes. Critical Care Medicine, 45, 58-68

25. Tejera, P., et al. (2020). Plasma levels of proresolving and prophlogistic lipid mediators: Association with severity of respiratory failure and mortality in acute respiratory distress syndrome. Critical Care Explorations, 2, e0241

26. Abdulnour, R. E., et al. (2018). Early intravascular events are associated with development of acute respiratory distress syndrome. A substudy of the LIPS-A clinical trial. American Journal of Respiratory and Critical Care Medicine, 197, 1575-1585

27. Zarbock, A., Polanowska-Grabowska, R. K., \& Ley, K. (2007). Platelet-neutrophil-interactions: linking hemostasis and inflammation. Blood Reviews, 21, 99-111

28. Looney, M. R., et al. (2009). Platelet depletion and aspirin treatment protect mice in a two-event model of transfusion-related acute lung injury. Journal of Clinical Investigation, 119, 3450-3461

29. Claria, J., \& Serhan, C. N. (1995). Aspirin triggers previously undescribed bioactive eicosanoids by human endothelial cellleukocyte interactions. Proceedings of the National Academy of Sciences of the United States of America, 92, 9475-9479

30. Ono, E., et al. (2014). Lipoxin generation is related to soluble epoxide hydrolase activity in severe asthma. American Journal of Respiratory and Critical Care Medicine, 190, 886-897

31. Dela Cruz, C. S., et al. (2018). Future research directions in pneumonia. NHLBI working group report. American Journal of Respiratory Critical Care Medicine, 198, 256-263

32. Balamayooran, G., Batra, S., Fessler, M. B., Happel, K. I., \& Jeyaseelan, S. (2010). Mechanisms of neutrophil accumulation in the lungs against bacteria. American Journal of Respiratory Cell and Molecular Biology, 43, 5-16

33. Seki, H., et al. (2010). The anti-inflammatory and proresolving mediator resolvin E1 protects mice from bacterial pneumonia and acute lung injury. Journal of Immunology, 184, 836-843

34. Flitter, B. A., et al. (2017). Pseudomonas aeruginosa sabotages the generation of host proresolving lipid mediators. Proceedings of the National Academy of Sciences of United States of America, $114,136-141$

35. Cohen, T. S., \& Prince, A. (2012). Cystic fibrosis: a mucosal immunodeficiency syndrome. Nature Medicine, 18, 509-519

36. Hvorecny, K. L., et al. (2018). An epoxide hydrolase secreted by Pseudomonas aeruginosa decreases mucociliary transport and hinders bacterial clearance from the lung. American Journal of Physiology Lung Cellular and Molecular Physiology, 314, L150-L156

37. Hudson, L. D., Milberg, J. A., Anardi, D., \& Maunder, R. J. (1995). Clinical risks for development of the acute respiratory distress syndrome. American Journal of Respiratory and Critical Care Medicine, 151, 293-301

38. Tavares, A. H., Colby, J. K., Levy, B. D., \& Abdulnour, R. E. (2019). A model of self-limited acute lung injury by unilateral intra-bronchial acid instillation. Journal of Visualized Experiments, https://doi.org/10.3791/60024
39. Levy, B. D., et al. (2011). The endogenous pro-resolving mediators lipoxin A4 and resolvin E1 preserve organ function in allograft rejection. Prostaglandins, Leukotrienes \& Essential Fatty Acids, 84, 43-50

40. Yipp, B. G., et al. (2012). Infection-induced NETosis is a dynamic process involving neutrophil multitasking in vivo. Nature Medicine, 18, 1386-1393

41. Arcanjo, A., et al. (2020). The emerging role of neutrophil extracellular traps in severe acute respiratory syndrome coronavirus 2 (COVID-19). Scientific Reports, 10, 19630

42. Krishnamoorthy, N., et al. (2018). Neutrophil cytoplasts induce TH17 differentiation and skew inflammation toward neutrophilia in severe asthma. Science Immunology, 3, eaao4747

43. Lachowicz-Scroggins, M. E., et al. (2019). Extracellular DNA, neutrophil extracellular traps, and inflammasome activation in severe asthma. American Journal of Respiratory and Critical Care Medicine, 199, 1076-1085

44. Levy, B. D., Clish, C. B., Schmidt, B., Gronert, K., \& Serhan, C. N. (2001). Lipid mediator class switching during acute inflammation: signals in resolution. Nature Immunology, 2, 612-619

45. Abdulnour, R. E., et al. (2016). Aspirin-triggered resolvin D1 is produced during self-resolving gram-negative bacterial pneumonia and regulates host immune responses for the resolution of lung inflammation. Mucosal Immunology, 9, 1278-1287

46. Loynes, C. A., et al. (2018). PGE2 production at sites of tissue injury promotes an anti-inflammatory neutrophil phenotype and determines the outcome of inflammation resolution in vivo. Science Advances, 4, eaar8320

47. Sham, H. P., et al. (2018). 15-epi-Lipoxin A4, resolvin D2, and resolvin D3 induce NF-kappaB regulators in bacterial pneumonia. Journal of Immunology, 200, 2757-2766

48. Fang, X., et al. (2015). Human mesenchymal stem (stromal) cells promote the resolution of acute lung injury in part through lipoxin A4. Journal of Immunology, 195, 875-881

49. Samuelsson, B., Dahlen, S. E., Lindgren, J. A., Rouzer, C. A., \& Serhan, C. N. (1987). Leukotrienes and lipoxins: structures, biosynthesis, and biological effects. Science, 237, 1171-1176

50. Levy, B. D., Petasis, N. A., \& Serhan, C. N. (1997). Polyisoprenyl phosphates in intracellular signalling. Nature, 389, 985-990

51. Levy, B. D., et al. (1999). Polyisoprenyl phosphate (PIPP) signaling regulates phospholipase D activity: a 'stop' signaling switch for aspirin-triggered lipoxin A4. The FASEB Journal, 13, 903-911

52. Levy, B. D., et al. (2005). Novel polyisoprenyl phosphates block phospholipase D and human neutrophil activation in vitro and murine peritoneal inflammation in vivo. British Journal of Pharmacology, 146, 344-351

53. Bonnans, C., Fukunaga, K., Keledjian, R., Petasis, N. A., \& Levy, B. D. (2006). Regulation of phosphatidylinositol 3-kinase by polyisoprenyl phosphates in neutrophil-mediated tissue injury. Journal of Experimental Medicine, 203, 857-863

54. Carlo, T., Kalwa, H., \& Levy, B. D. (2013). 15-Epi-lipoxin A4 inhibits human neutrophil superoxide anion generation by regulating polyisoprenyl diphosphate phosphatase 1. The FASEB Journal, 27, 2733-2741

55. Carlo, T., Petasis, N. A., \& Levy, B. D. (2009). Activation of polyisoprenyl diphosphate phosphatase 1 remodels cellular presqualene diphosphate. Biochemistry, 48, 2997-3004

56. Fukunaga, K., et al. (2006). Identification and functional characterization of a presqualene diphosphate phosphatase. Journal of Biological Chemistry, 281, 9490-9497

57. Miriyala, S., et al. (2010). Functional characterization of the atypical integral membrane lipid phosphatase PDP1/PPAPDC2 identifies a pathway for interconversion of isoprenols and isoprenoid phosphates in mammalian cells. Journal of Biology Chemistry, 285, 13918-13929 
58. Carlo, T., \& Levy, B. D. (2008). Chemical mediators and the resolution of airway inflammation. Allergology International, 57, 299-305

59. Cummings, R. J., et al. (2002). Phospholipase D activation by sphingosine 1-phosphate regulates interleukin-8 secretion in human bronchial epithelial cells. Journal of Biology Chemistry, 277, 30227-30235

60. Hewitt, R. J. \& Lloyd, C. M. (2021). Regulation of immune responses by the airway epithelial cell landscape. Nature Review Immunology, 6, 347-362

61. Abdulnour, R. E., et al. (2018). Phospholipase D isoforms differentially regulate leukocyte responses to acute lung injury. Journal of Leukocyte Biology, 103, 919-932

62. Chollet-Martin, S., et al. (1993). High levels of interleukin-8 in the blood and alveolar spaces of patients with pneumonia and adult respiratory distress syndrome. Infection and Immunity, 61, $4553-4559$

63. Natarajan, V., Jayaram, H. N., Scribner, W. M., \& Garcia, J. G. (1994). Activation of endothelial cell phospholipase D by sphingosine and sphingosine-1-phosphate. American Journal of Respiratory Cell and Molecular Biology, 11, 221-229

64. Chen, Q., et al. (2020). Angiocrine sphingosine-1-phosphate activation of S1PR2-YAP signaling axis in alveolar type II cells is essential for lung repair. Cell Reports, 31, 107828

65. Fu, P., et al. (2021). NOX4 mediates pseudomonas aeruginosainduced nuclear reactive oxygen species generation and chromatin remodeling in lung epithelium. Antioxidants (Basel), 10, 477

66. Suryadevara, V., et al. (2018). Sphingolipids in ventilator induced lung injury: role of sphingosine-1-phosphate lyase. International Journal of Molecular Sciences, 19, 114

67. Suryadevara, V., Ramchandran, R., Kamp, D. W. \& Natarajan, V. (2020). Lipid mediators regulate pulmonary fibrosis: potential mechanisms and signaling pathways. International Journal of Molecular Sciences, 21, 4257

68. Ebenezer, D. L., et al. (2020). S1P and plasmalogen derived fatty aldehydes in cellular signaling and functions. Biochimica et Biophysica Acta Molecular and Cell Biology of Lipids, 1865, 158681

69. Ebenezer, D. L., et al. (2019). Genetic deletion of Sphk2 confers protection against Pseudomonas aeruginosa mediated differential expression of genes related to virulent infection and inflammation in mouse lung. BMC Genomics, 20, 984

70. Ebenezer, D. L., Fu, P., Suryadevara, V., Zhao, Y., \& Natarajan, V. (2017). Epigenetic regulation of pro-inflammatory cytokine secretion by sphingosine 1-phosphate (S1P) in acute lung injury: Role of S1P lyase. Advances in Biological Regulation, 63, 156-166

71. Ebenezer, D. L., Fu, P., \& Natarajan, V. (2016). Targeting sphingosine-1-phosphate signaling in lung diseases. Pharmacology and Therapeutics, 168, 143-157

72. Usatyuk, P. V., Kotha, S. R., Parinandi, N. L., \& Natarajan, V. (2013). Phospholipase D signaling mediates reactive oxygen species-induced lung endothelial barrier dysfunction. Pulmonary Circulation, 3, 108-115

73. Bonnans, C., \& Levy, B. D. (2007). Lipid mediators as agonists for the resolution of acute lung inflammation and injury. American Journal of Respiratory Cell and Molecular Biology, 36, 201-205

74. Sanak, M., et al. (2000). Aspirin-tolerant asthmatics generate more lipoxins than aspirin-intolerant asthmatics. European Respiratory Journal, 16, 44-49

75. Levy, B. D., et al. (2005). Diminished lipoxin biosynthesis in severe asthma. American Journal of Respiratory and Critical Care Medicine, 172, 824-830
76. Barnig, C., et al. (2013). Lipoxin A4 regulates natural killer cell and type 2 innate lymphoid cell activation in asthma. Science Translational Medicine, 5, 174ra126

77. Urbach, V., Higgins, G., Buchanan, P., \& Ringholz, F. (2013). The role of Lipoxin A4 in cystic fibrosis lung disease. Computational Structural Biotechnology Journal, 6, e201303018

78. Bozinovski, S., Anthony, D., \& Vlahos, R. (2014). Targeting proresolution pathways to combat chronic inflammation in COPD. Journal of Thoracic Disease, 6, 1548-1556

79. Jin, S. W., et al. (2007). Posttreatment with aspirin-triggered lipoxin A4 analog attenuates lipopolysaccharide-induced acute lung injury in mice: the role of heme oxygenase-1. Anesthesia and Analgesia, 104, 369-377

80. Haworth, O., Cernadas, M., Yang, R., Serhan, C. N., \& Levy, B. D. (2008). Resolvin E1 regulates interleukin 23, interferon-gamma and lipoxin A4 to promote the resolution of allergic airway inflammation. Nature Immunology, 9, 873-879

81. El Kebir, D., Gjorstrup, P., \& Filep, J. G. (2012). Resolvin E1 promotes phagocytosis-induced neutrophil apoptosis and accelerates resolution of pulmonary inflammation. Proceedings of the National Academy Sciences of United States of America, 109, 14983-14988

82. Hisada, T., Ishizuka, T., Aoki, H., \& Mori, M. (2009). Resolvin E1 as a novel agent for the treatment of asthma. Expert Opinion on Therapeutic Targets, 13, 513-522

83. Xia, H., et al. (2019). Resolvin D1 alleviates ventilator-induced lung injury in mice by activating PPARgamma/NF-kappaB signaling pathway. Biomed Research International, 2019, 6254587

84. Zhang, H. W., et al. (2019). RvD1 ameliorates LPS-induced acute lung injury via the suppression of neutrophil infiltration by reducing CXCL2 expression and release from resident alveolar macrophages. International Immunopharmacology, 76, 105877

85. Chiang, N., de la Rosa, X., Libreros, S., \& Serhan, C. N. (2017). Novel resolvin D2 receptor axis in infectious inflammation. Journal of Immunology, 198, 842-851

86. Levy, B. D., et al. (2007). Protectin D1 is generated in asthma and dampens airway inflammation and hyperresponsiveness. Journal of Immunology, 178, 496-502

87. Miyata, J., et al. (2013). Dysregulated synthesis of protectin D1 in eosinophils from patients with severe asthma. The Journal of Allergy Clinical Immunology, 131, 353-360

88. Li, X., et al. (2014). Protectin D1 promotes resolution of inflammation in a murine model of lipopolysaccharide-induced acute lung injury via enhancing neutrophil apoptosis. Chinese Medical Journal, 127, 810-814

89. Freedman, S. D., et al. (2004). Association of cystic fibrosis with abnormalities in fatty acid metabolism. The New England Journal of Medicine, 350, 560-569

90. Serhan, C. N., et al. (2009). Maresins: novel macrophage mediators with potent antiinflammatory and proresolving actions. Journal of Experimental Medicine, 206, 15-23

91. Ou, G., et al. (2021). The protective effects of Maresin 1 in the OVA-induced asthma mouse model. Mediators of Inflammation, 2021, 4131420

92. Qiao, N., et al. (2020). Maresin1 promotes M2 macrophage polarization through peroxisome proliferator-activated receptorgamma activation to expedite resolution of acute lung injury. Journal of Surgical Research, 256, 584-594

93. Nordgren, T. M., et al. (2015). Maresin-1 reduces airway inflammation associated with acute and repetitive exposures to organic dust. Translational Research, 166, 57-69 